\section{Prevalência de fraturas vertebrais e fatores de risco em mulheres com mais de 60 anos de idade na cidade de Chapecó, Santa Catarina, Brasil}

\author{
Prevalence of vertebral fractures and risk factors \\ in women over 60 years of age in Chapecó, \\ Santa Catarina State, Brazil
}

\author{
1 Instituto Fernandes \\ Figueira, Fundação Oswaldo \\ Cruz, Rio de Janeiro, Brasil. \\ 2 Universidade Comunitária \\ da Região de Chapecó, \\ Chapecó, Brasil. \\ 3 Faculdade de Medicina, \\ Universidade Federal do Rio \\ Grande do Sul, Porto Alegre, \\ Brasil. \\ 4 Hospital Unimed, Chapecó, \\ Brasil. \\ 5 Faculdade de Medicina \\ Universidade do Estado do \\ Rio de Janeiro, Rio de Janeiro, \\ Brasil. \\ Correspondência \\ P. P. Oliveira \\ Instituto Fernandes Figueira, \\ Fundação Oswaldo Cruz. \\ Rua Senador Atílio Fontana, \\ 591-E, Chapecó, SC \\ 89809-000, Brasil. \\ patriciapoliveira@hotmail.com
}

\author{
Patricia Pereira de Oliveira 1,2 \\ Lizanka Paola Figueiredo Marinheiro ${ }^{1}$ \\ Maria Celeste Osório Wender 3 \\ Felipe Roisenberg 4 \\ Paulo Gustavo Sampaio Lacativa 5
}

\section{Introdução}

O aumento na ocorrência de fraturas secundárias à fragilidade óssea representa um significativo problema de Saúde Pública, já que corresponde a um importante aumento na morbidade, mortalidade e nos custos 1 . A fratura de coluna vertebral tende a ser a manifestação mais comum e precoce relacionada a essa fragilidade em populações de mulheres com idade superior a 45 anos. Ocorre principalmente na junção da coluna torácica e lombar e na área média do tórax ${ }^{2}$, representando um risco em torno de $20 \%$ a mais de ocorrência de nova fratura vertebral 3 , fratura de quadril e de antebraço ${ }^{4}$. Uma vez que apenas $30 \%$ das fraturas se manifestam como dor nas costas e a solicitação de radiografia de coluna não é feita rotineiramente para rastreamento, estima-se que somente $25 \%$ das fraturas vertebrais sejam diagnosticadas 5. São ainda associadas à hipercifose, à redução da altura e à limitação de movimento, o que pode levar à necessidade de auxílio nas atividades rotineiras, reduzindo a qualidade de vida e aumentando a mortalidade $2,4,6,7$.

O paciente com fratura vertebral tem o risco de novas fraturas de coluna aumentado em até 25 vezes se houver queda na densidade mineral óssea em exames subseqüentes, mesmo mantendo-se dentro da faixa de normalidade 5 . Apesar do uso de instrumentos clínicos para rastrear mulheres com risco de baixa densidade mineral óssea, nenhum deles ainda é capaz de 
identificar, com precisão, as que estão em risco de fratura 8,9,10. Porém, a avaliação conjunta de exames complementares (densitometria, ultrassonometria, tomografia quantitativa, entre outros) e fatores clínicos voltados para a população de cada país ou região parece ser uma idéia promissora 11.

Os fatores de risco para osteoporose e para fraturas se confundem, destacando-se o sexo feminino, fratura prévia, idade avançada, menopausa precoce e não tratada, além de terapia com corticóides ${ }^{12}$. As mulheres brancas, na pós-menopausa, apresentam maior incidência de fraturas; a partir dos 50 anos, 30\% das mulheres poderão sofrer algum tipo de fratura por fragilidade ao longo da vida 13. Estudos realizados no Brasil mostram variações regionais, provavelmente explicadas pelas características de cada população, situação geográfica do território onde reside e fatores ambientais 11,13. Nestes estudos, a prevalência de fraturas vertebrais variou entre $14,8 \%$ e $36,8 \% 7,14,15$; a incidência de fraturas de quadril, entre 5,59 e 27,7/10.000 habitantes (sendo a maior taxa referente à população feminina) $13,16,17$.

O presente estudo teve como objetivo verificar a ocorrência de fratura vertebral assintomática por fragilidade óssea em uma população de mulheres brasileiras na pós-menopausa residentes no Sul do país e correlacionar os achados com dados clínicos, demográficos e comportamentais.

\section{Materiais e métodos}

Foi realizado estudo epidemiológico de corte transversal na cidade de Chapecó, Santa Catarina. Chapecó é uma cidade de colonização italiana, alemã e polonesa (Secretaria do Desenvolvimento Econômico e Agricultura do Município de Chapecó. Banco de dados. Boletim Informativo 2004), localizada ao Sul do Brasil (latitude $-27^{\circ} 5^{\prime} 47^{\prime \prime}$, longitude $\left.52^{\circ} 37^{\prime} 6^{\prime \prime}\right)$, com população estimada de 173.261 habitantes, $6,14 \%$ dos quais são pessoas com idade superior a 60 anos. Possui renda per capita de 21,22 salários mínimos e índice de desenvolvimento humano (IDH) de 0,848, ambos acima do que se encontra na maioria dos municípios brasileiros (Departamento de Informática do SUS. População residente - Santa Catarina. http://tabnet.datasus.gov.br/cgi/tabcgi. exe?ibge/cnv/popSC.def, acessado em 04/ Abr/2006).

Selecionou-se uma amostragem aleatória estratificada por bairro de origem da população feminina acima de 60 anos residentes no município. O tamanho amostral de 190 mulheres foi calculado adotando-se uma prevalência provável do evento (fratura) de $15 \% 18$, nível de $95 \%$ de confiança, e erro tolerável de $5 \%$, para população potencialmente elegível de 5.781 mulheres (correspondendo ao número total de mulheres com idade igual ou superior a 60 anos residentes na cidade, no ano de 2006, segundo dados do Departamento de Informática do SUS - DATASUS). Optamos por aumentar a amostra em, no mínimo, $25 \%$ para manter o seu poder, mesmo com possíveis perdas.

Chapecó conta com vinte unidades básicas de saúde (UBS) distribuídas ao longo de seu território, as quais têm 5.162 mulheres com idade acima de 60 anos cadastradas, correspondendo a $87,92 \%$ da população feminina residente no município com essa mesma faixa etária (Departamento de Informática do SUS. População residente - Santa Catarina. http://tabnet.datasus. gov.br/cgi/tabcgi.exe?ibge/cnv/popSC.def, acessado em 04/Abr/2006). Logo, utilizamos o cadastro das UBS para selecionar a amostra por este ser representativo da população local. Considerando as características das áreas abrangidas pelas UBS e o número de pessoas atendidas em cada uma, optamos por estratificar a população de acordo com o nível social de cada bairro de residência a fim de evitar viés de seleção. Cada UBS foi distribuída, segundo o bairro de origem, em classe baixa ou alta, conforme classificação atual adotada pela Secretaria Municipal de Saúde e pela Fundação de Amparo Social, com alocação proporcional das mulheres. Em seguida, realizamos sorteio com base no cadastro feito na UBS.

O contato inicial para inclusão no estudo foi realizado pelo pesquisador principal. A coleta de dados foi feita por meio de questionário estruturado, aplicado ao longo de visitas domiciliares por uma equipe de estudantes de medicina previamente treinados e após estudo-piloto, entre os meses de maio e dezembro de 2007. Após o questionário, as pacientes foram encaminhadas para realização de radiografia de coluna vertebral (RX) para avaliação de fraturas.

Os critérios de inclusão foram: sexo feminino; cor branca (auto-referida); idade superior a 60 anos; diagnóstico clínico de menopausa (ausência de menstruação por pelo menos um ano) e residência fixa na cidade de Chapecó. Os critérios de exclusão foram: prejuízo cognitivo importante que impossibilitasse a obtenção das respostas do questionário; história pessoal de doenças que sabidamente afetam o metabolismo ósseo (como artrite reumatóide, lúpus eritematoso sistêmico, hiperparatireoidismo, osteogênese imperfecta); neoplasias malignas nos últimos cinco anos, exceto carcinoma basocelular de pele.

Para avaliação de fatores relacionados à fragilidade óssea e à deformidade vertebral, utilizou- 
se questionário conhecido como EVOS (European Vertebral Osteoporosis Study), já traduzido e validado para 14 línguas 19, anteriormente utilizado em importantes estudos nacionais 7,14. O questionário é composto pelos seguintes dados: antropometria, história médica pessoal prévia e atual, história familiar, uso de medicações com ação sobre o metabolismo ósseo, hábitos de vida, impacto da doença sobre a saúde geral do indivíduo. Os dados antropométricos foram aferidos segundo critérios adotados internacionalmente, com medidas de peso e altura observando intervalos de $0,1 \mathrm{~kg}$ e $0,01 \mathrm{~m}$, respectivamente. $\mathrm{O}$ índice de massa corporal (IMC) foi calculado pela fórmula peso/altura $2\left(\mathrm{~kg} / \mathrm{m}^{2}\right)$ e classificado conforme critérios da Organização Mundial da Saúde (OMS) para obesidade 20. Para cálculo aproximado da ingestão alimentar diária de cálcio, utilizou-se uma adaptação da Tabela Brasileira de Composição de Alimentos 21: 1 fatia queijo (90mg); 1 copo de iogurte $(121,2 \mathrm{mg})$; 1 copo leite ( $257 \mathrm{mg})$; 1 bola se sorvete (135mg). O nível de atividade física foi classificado em: nível 1 - atividade leve (trabalho burocrático em escritório, na maior parte do tempo sentado); nível 2 atividade moderada (trabalhos na maior parte do tempo em pé, como vendedor e dona-de-casa); nível 3 - atividade pesada (enfermeira, empregada doméstica); nível 4 - atividade muito pesada (agricultor, esportista, pedreiro) 19 .

A aferição da circunferência da cintura foi tomada na menor curvatura localizada entre as costelas e a crista ilíaca (cintura natural do indivíduo); a da circunferência do quadril, na extensão máxima das nádegas (protuberância glútea). As medidas foram feitas com o indivíduo em pé, em posição ereta, abdômen relaxado, braços ao lado do corpo e os pés juntos, utilizando-se uma fita métrica flexível e inextensível de $200 \mathrm{~cm}$ de comprimento, com precisão de uma casa decimal. A leitura foi feita no centímetro mais próximo, no ponto de cruzamento da fita. A relação cintura/ quadril foi então calculada por divisão simples destas medidas 22 .

As radiografias de coluna vertebral torácica e lombar em perfil foram realizadas em um mesmo Serviço de Radiologia, dentro das normas do programa de qualidade do Colégio Brasileiro de Radiologia. A aquisição das imagens obedeceu aos critérios padronizados internacionalmente quanto a técnica, tamanho do filme, tempo de exposição, pico de $\mathrm{kV}$, colimação da fonte $\mathrm{RX}$, posicionamento do paciente, distância do foco e momento da respiração. As radiografias foram avaliadas, de forma "cega" e individualizada, por radiologista com titulo de especialista e experiência por tempo superior a dois anos. O profissional foi orientado a fazer avaliação da coluna torácica e lombar (T4-L5) para fraturas vertebrais, segundo o método semiquantitativo proposto por Genant et al. 23. Esse método consiste na determinação visual da extensão da redução da altura da vértebra, sem medidas diretas, sendo classificada em: grau 0 (normal); grau 1 (deformidade leve: 20\%-25\% de redução na altura anterior, média ou posterior, e 10\%-20\% de redução da área vertebral projetada); grau 2 (deformidade moderada: 25\%-40\% de redução na altura anterior, média ou posterior, e $20 \%-40 \%$ de redução da área vertebral projetada); grau 3 (deformidade severa: $40 \%$ ou mais de redução na altura anterior, média ou posterior e na área vertebral projetada). Foram consideradas fraturadas as vértebras que receberam graduação $\geq 1$. Para cada vértebra, utilizamos avaliação binária (sim ou não) quanto à presença de fratura e, em caso afirmativo, classificação conforme o tipo de fratura (cunha, bicôncava ou esmagamento). Outras deformidades não relacionadas à fratura não foram avaliadas. Foi calculado o índice kappa como verificação de concordância entre dois observadores com as mesmas características descritas anteriormente, com resultado de 0,89 para coluna torácica e lombar.

As variáveis independentes incluídas na análise foram: faixa etária (60-69 anos; 70-79 anos; mais de 80 anos), história familiar de fratura de quadril para parentes de primeiro grau (sim ou não), história prévia pessoal de fratura (sim ou não), IMC (sobrepeso/obeso, normal/abaixo do normal), uso de terapia hormonal por mais de um ano (sim ou não), uso de suplementos de cálcio (atual, no passado ou nunca), tabagismo (atual, no passado ou nunca), ingestão de álcool (nunca ou regularmente, independentemente da freqüência), atividade física atual (por tempo superior a 30 minutos ou nunca), ingestão de cálcio alimentar diário (maior ou menor que 300mg). Na análise bruta, a prevalência de fraturas foi calculada para cada grupo das variáveis independentes e, na análise ajustada, foi realizada regressão de Poisson, com variância robusta, obtendo-se razões de prevalência ajustadas, com intervalos de 95\% de confiança (IC95\%). Neste modelo, foram incluídas apenas as variáveis com nível de $\mathrm{p}<0,20$ na análise bruta. A análise estatística foi realizada com programa SPSS versão 10.0 (SPSS Inc., Chicago, Estados Unidos).

Todas as mulheres assinaram o Termo de Consentimento Livre e Esclarecido antes de qualquer procedimento. Este estudo obedeceu aos critérios de ética preconizados pela Resolução $n^{o}$. 196/96 do Conselho Nacional de Saúde do Ministério da Saúde, tendo sido previamente registrado sob no. 101/06 e aprovado pelo Comitê de Ética em Pesquisa da Universidade Comuni- 
tária da Região de Chapecó, não apresentando conflito de interesses.

\section{Resultados}

Em um primeiro momento, foram entrevistadas 300 mulheres, 66 das quais foram excluídas por não preencherem os critérios de inclusão $(68,2 \%)$, não desejarem participar $(15,1 \%)$, ou não serem encontradas após duas visitas domiciliares (16,7\%). Das 234 mulheres elegíveis inicialmente contatadas, 186 (79,5\%) fizeram o protocolo completo. As desistentes $(n=48)$ não quiseram comparecer para realização do exame, mesmo após serem recontatadas. As características antropométricas e reprodutivas das participantes estão expressas na Tabela 1. A idade da população estudada variou entre 60 e 91 anos, e $52,2 \%$ pertenciam a bairros de classe alta. Não houve diferença estatisticamente significativa entre mulheres pertencentes a bairros de diferentes classes quanto às características descritas na Tabela 1 .

Tabela 1

Características antropométricas e reprodutivas das mulheres da população de estudo $(\mathrm{N}=186)$. Chapecó, Santa Catarina, Brasil.

\begin{tabular}{lc}
\hline Características & Média \pm desvio-padrão \\
\hline Idade (anos) & $69,61 \pm 6,30$ \\
Idade da menarca (anos) & $13,65+1,85$ \\
Idade da menopausa (anos) & $48,64 \pm 5,68$ \\
Tempo de menopausa (anos) & $21,61 \pm 9,40$ \\
Peso $(\mathrm{kg})$ * & $68,02 \pm 14,00$ \\
Altura $(\mathrm{m}){ }^{*}$ & $1,56 \pm 0,06$ \\
IMC $\left(\mathrm{kg} / \mathrm{m}^{2}\right)$ * & $27,95 \pm 5,24$ \\
Relação cintura/quadril * & $0,92 \pm 0,16$
\end{tabular}

IMC: índice de massa corporal.

${ }^{*} \mathrm{n}=152$.
$1,6 \%(\mathrm{n}=3)$ tinha baixo peso; $14,5 \%(\mathrm{n}=27)$ eram tabagistas atuais e $33,3 \%(n=62)$ eram sedentárias. A maioria negou uso regular de bebidas alcoólicas $(62,4 \%)$, e ninguém referiu consumo alimentar diário de cálcio acima de $600 \mathrm{mg}$.

Do total de 2.604 vértebras analisadas, 156 (6\%) estavam fraturadas. Quase metade das mulheres avaliadas $(48,9 \%)$ tinha, no mínimo, uma fratura vertebral não associada à história prévia de fratura por alto impacto; $31,7 \%(n=59)$ tinham fraturas em vértebras torácicas; $25,8 \%$, $(n=48)$, em vértebras lombares; e $8,6 \%(n=16)$ tinham fraturas em ambos os sítios. A maior prevalência de fraturas por vértebra foi observada no final da coluna torácica (T11 e T12) e lombar (L4-L5) (Figura 1). Observou-se maior freqüência de fratura por esmagamento na base da coluna vertebral e de fratura em cunha na área média (T6-7) e final (T11-12) do tórax, conforme a Figura 2.

A Tabela 2 apresenta os resultados da análise bruta da razão de prevalências de acordo com as categorias dos fatores de risco para fraturas; na Tabela 3, verificam-se os resultados da análise ajustada. Observa-se que existe gradiente entre idade e fratura vertebral, chegando a ser 2,4 vezes maior a prevalência de fraturas entre as mulheres com idade superior a 80 anos. As sedentárias apresentaram prevalência de fratura 1,57 vezes maior do que as não sedentárias. Na análise ajustada, o ajuste foi feito apenas pelas variáveis que, no modelo anterior (Tabela 2), apresentaram associação com fratura vertebral em nível de $\mathrm{p}$ $<0,20$. Optou-se por não se utilizar ajuste pelo tempo de menopausa em virtude da colinearidade com a idade, mesmo assim o sedentarismo e a idade foram os únicos fatores de risco que apresentaram significância estatística após ajuste.

\section{Discussão}

Ainda existem poucos estudos brasileiros sobre a prevalência de fraturas vertebrais. A dimensão do território e as diferenças regionais dificultam extrapolar os resultados para toda a população residente no país. Encontramos uma alta prevalência de fraturas na coluna, sendo T11-12 e L4-5 os sítios mais envolvidos. Essa prevalência aumentou com o avanço da idade. As mulheres com evidência radiográfica de fratura vertebral tinham maior tempo de menopausa do que as sem fraturas, possivelmente por aquelas serem mais velhas. Na análise (razão de prevalências ajustada), o sedentarismo e a idade mostraram-se como fatores de risco com significância estatística.

Comparando os resultados de nosso estudo (população de amostra oriunda da cidade de 
Figura 1

Tipo de fratura por vértebra. Chapecó, Santa Catarina, Brasil.

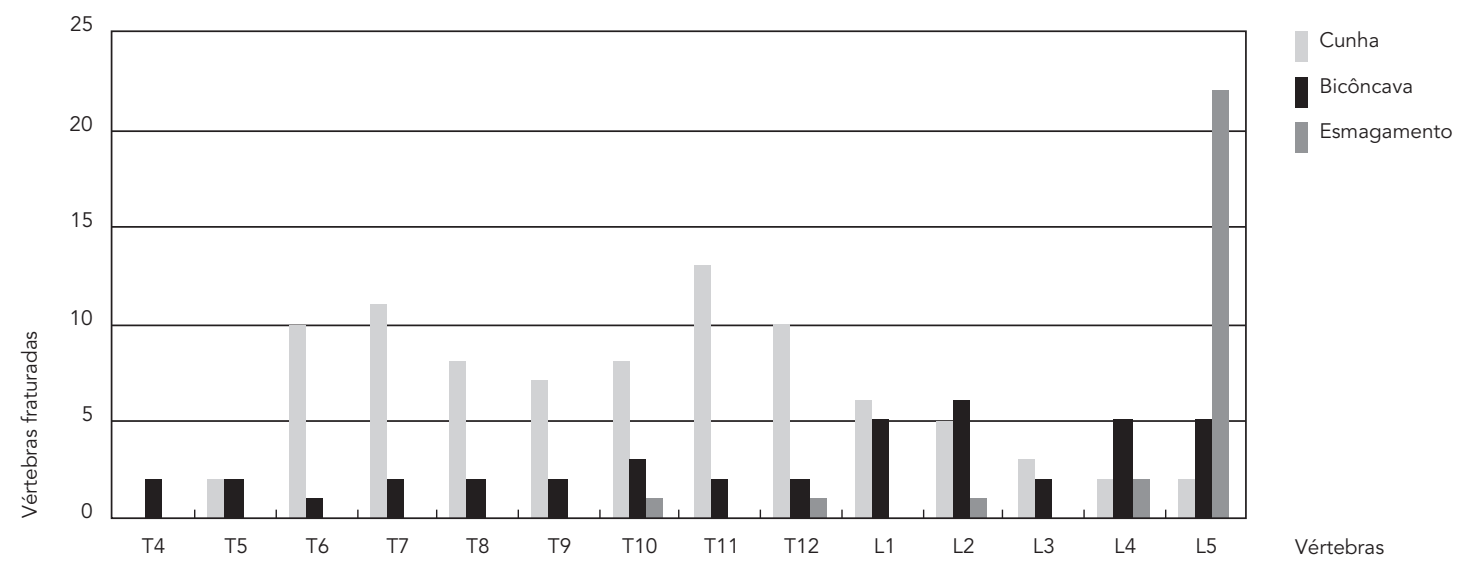

Figura 2

Frequência de fratura por vértebra. Chapecó, Santa Catarina, Brasil.

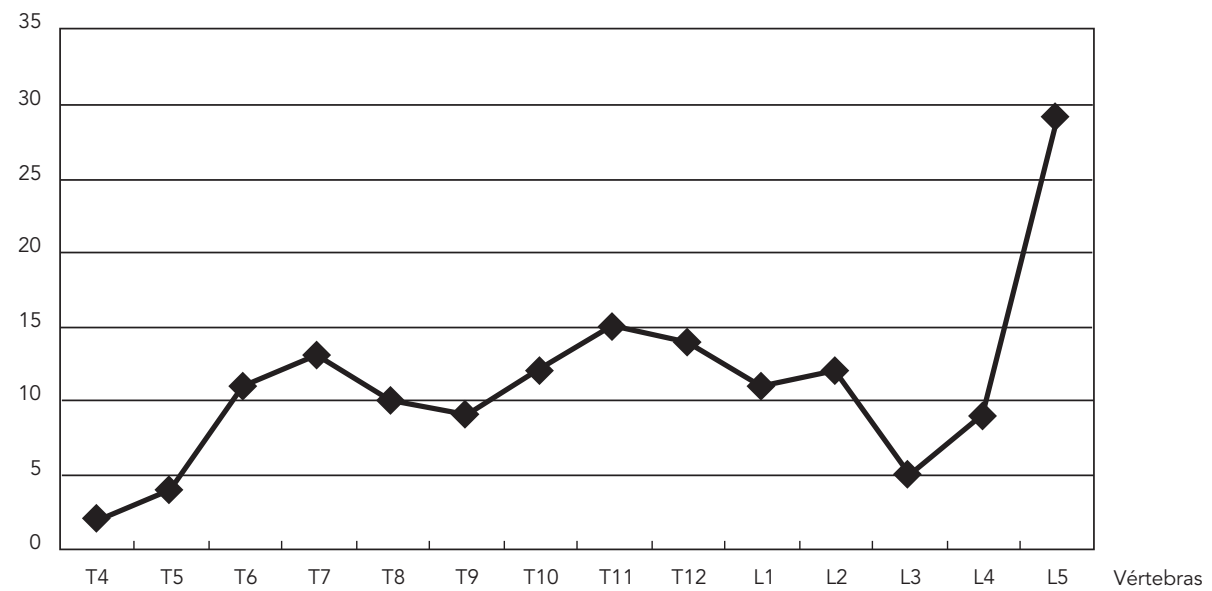

Chapecó, no Sul do país) com os de outras investigações brasileiras, encontramos dados divergentes. O estudo conhecido como LAVOS 14, cuja população de origem era de mulheres residentes em Vitória, no Espírito Santo, na Região Sudeste do Brasil, demonstrou uma prevalência de fratura vertebral de $14,2 \%$, praticamente um terço da nossa. Essa divergência poderia ser atribuída primeiramente à diferença nos critérios de definição e análise das fraturas. No trabalho capixaba, optou-se pela medida direta da vértebra e análise de T4 a L4, enquanto, no presente estudo, optamos por análise visual de T4 a L5. Porém, embora tenhamos encontrado alta prevalência de fraturas em L5, se excluíssemos da análise de prevalência as 13 mulheres com fratura exclusiva 
Tabela 2

Prevalência de fratura vertebral (\%) e razões de prevalência (RP) segundo categorias de fatores de risco (N=186). Chapecó, Santa Catarina, Brasil.

\begin{tabular}{|c|c|c|c|c|}
\hline Fatores de risco & Com fratura/n & $\%$ & RP bruta (IC95\%) & Valor de $p$ \\
\hline \multicolumn{5}{|l|}{ Faixa etária (anos) } \\
\hline $60-69$ & $36 / 99$ & 36,4 & 1,00 & \\
\hline $70-79$ & $42 / 72$ & 58,3 & $1,60(1,16-2,22)$ & $<0,05$ \\
\hline$>80$ & 13/15 & 86,7 & $2,38(1,72-3,31)$ & $<0,001$ \\
\hline \multicolumn{5}{|c|}{ História familiar de fratura de quadril } \\
\hline Não & $77 / 151$ & 51,0 & 1,00 & \\
\hline Sim & $11 / 26$ & 42,4 & $0,83(0,52-1,34)$ & 0,44 \\
\hline \multicolumn{5}{|l|}{ História prévia de fratura } \\
\hline Não & $66 / 141$ & 46,8 & 1,00 & \\
\hline Sim & $25 / 45$ & 55,6 & $1,19(0,87-1,63)$ & 0,29 \\
\hline \multicolumn{5}{|l|}{$\mathrm{IMC}$ * } \\
\hline Sobrepeso/Obesidade & $45 / 98$ & 45,8 & 1,00 & \\
\hline Normal/Baixo & $31 / 54$ & 57,4 & $0,80(0,58-1,10)$ & 0,16 \\
\hline \multicolumn{5}{|c|}{ Uso de terapia hormonal > 1 ano } \\
\hline Sim & $21 / 46$ & 45,7 & 1,00 & \\
\hline Não & $12 / 25$ & 48,0 & $1,05(0,63-1,76)$ & 0,85 \\
\hline \multicolumn{5}{|c|}{ Uso de suplemento de cálcio } \\
\hline Atual & $33 / 69$ & 36,7 & 1,00 & \\
\hline Nunca & $53 / 108$ & 57,8 & $1,02(0,74-1,39)$ & 0,92 \\
\hline No passado & $5 / 9$ & 55,6 & $1,16(0,62-2,19)$ & 0,64 \\
\hline \multicolumn{5}{|l|}{ Tabagismo } \\
\hline Nunca & 49/110 & 44,5 & 1,00 & \\
\hline No passado & $25 / 49$ & 51,0 & $1,15(0,81-1,62)$ & 0,44 \\
\hline Atual & $17 / 27$ & 63,0 & $1,41(0,99-2,02)$ & 0,06 \\
\hline \multicolumn{5}{|l|}{ Ingesta de álcool } \\
\hline Nunca & $60 / 116$ & 51,7 & 1,00 & \\
\hline Regularmente & $31 / 70$ & 44,3 & $0,86(0,62-1,18)$ & 0,34 \\
\hline \multicolumn{5}{|l|}{ Atividade física atual } \\
\hline$\geq 30$ minutos/dia & $51 / 124$ & 41,1 & 1,00 & \\
\hline Nenhuma & $40 / 62$ & 64,5 & $1,57(1,19-2,08)$ & 0,02 \\
\hline \multicolumn{5}{|c|}{ Ingesta de cálcio alimentar diário } \\
\hline$>300 \mathrm{mg}$ & $40 / 82$ & 48,0 & 1,00 & \\
\hline$\leq 300 \mathrm{mg}$ & $51 / 104$ & 49,0 & $1,01(0,75-1,35)$ & 0,97 \\
\hline
\end{tabular}

IMC: índice de massa corporal.

${ }^{*} \mathrm{n}=152$.

da L5, contaríamos, ainda, com uma prevalência de fraturas de $41,9 \%$, valor superior ao encontrado em quaisquer estudos nacionais.

Também se pode atribuir essa discordância às diferenças entre as populações estudadas. Ainda que os dois municípios possuam IDH e renda per capita semelhantes, o que por si só já destoa das outras cidades da União, as características gerais de ambos são diferentes. Por exemplo, quanto à cor da pele, embora $54 \%$ da população brasileira se auto-refira como branca, há variação de $28 \%$ no Norte a $83 \%$ no Sul do país 24 ; logo, a cor da pele pode ser um dos fatores responsáveis pe- la maior prevalência de fraturas na população analisada neste estudo. Nele, utilizamos a cor não-branca como fator de exclusão para evitar viés, porém apenas quatro mulheres foram excluídas da amostra por esse motivo. Ainda que não descrito na investigação capixaba, a região de origem da amostra do LAVOS possui $64 \%$ da população referida como branca, segundo dados do Instituto Brasileiro de Geografia e Estatística (IBGE) 24 .

Outros estudos brasileiros também reforçam essas variações regionais, como o de Bandeira \& Carvalho 15 , com $36,8 \%$ de fraturas de coluna 
Tabela 3

Razões de prevalência (RP) ajustadas (N = 186). Chapecó, Santa Catarina, Brasil.

\begin{tabular}{|c|c|c|}
\hline Fatores de risco & RP ajustada (IC95\%) & Valor de $p$ \\
\hline \multicolumn{3}{|l|}{ Faixa etária (anos) } \\
\hline $60-69$ & 1,00 & \\
\hline $70-79$ & $1,72(1,20-2,48)$ & 0,003 \\
\hline$>80$ & $2,33(1,63-3,33)$ & $<0,001$ \\
\hline \multicolumn{3}{|l|}{$\mathrm{IMC}$ * } \\
\hline Normal & 1,00 & \\
\hline Sobrepeso & $0,92(0,69-1,22)$ & 0,562 \\
\hline \multicolumn{3}{|l|}{ Tabagismo } \\
\hline Nunca & 1,00 & \\
\hline No passado & $1,05(0,73-1,50)$ & 0,812 \\
\hline Atual & $1,44(0,97-2,14)$ & 0,072 \\
\hline \multicolumn{3}{|l|}{ Atividade física atual } \\
\hline$\geq 30$ minutos/dia & 1,00 & \\
\hline Nenhuma & $1,44(1,07-1,95)$ & 0,016 \\
\hline
\end{tabular}

IMC: índice de massa corporal.

${ }^{*} \mathrm{n}=152$.

em uma população de mulheres de Pernambuco (Região Nordeste), e Pinheiro et al. 7, com 27,2\% entre mulheres residentes em São Paulo (Região Sudeste). Ambos os trabalhos utilizaram medidas diretas para definição de fraturas de T4 a L5. O estudo conhecido como BRAZOS 11 identificou uma freqüência variável de fraturas por fragilidade (auto-referidas) de diferentes sítios nas cinco regiões geográficas do país, tendo variado de $10,5 \%$ na Região Centro-oeste a $16,2 \%$ na Região Sul entre a população feminina. Quando comparadas todas essas regiões, observam-se diferenças quanto à cor da pele e hábitos de vida, como tabagismo, grau de atividade física, consumo de álcool, IMC, entre outros, todos esses fatores com influência já descrita em alguns estudos nacionais 7,25 sobre a massa óssea e, provavelmente, sobre a resistência a fraturas. Variações regionais também são descritas no continente europeu, com maior prevalência de fraturas vertebrais na Suécia $(27,8 \%)$ e menor em algumas cidades do Sul e nos países mediterrâneos (14,9\% em Madri, $15,9 \%$ na Turquia) 26 .

Em adição às características do grupo analisado neste estudo, outra explicação para essas variações regionais é a situação geográfica. Trata-se de uma população que, além de ser predominantemente branca, reside em um território ao Sul do país, a $27^{\circ}$ abaixo da linha do Equador (670m do nível do mar, latitude -275’47”), o que poderia por si só denotar aumento de risco para fraturas, conforme sugerido por Johnell et al. 27. Esses autores observaram aumento na fre- qüência de fraturas a cada $10^{\circ}$ de afastamento da linha do Equador, achado ratificado por Castro da Rocha \& Ribeiro 13 ao encontrarem, na cidade de Sobral, Ceará, incidência de fraturas do colo do fêmur quatro vezes menor do que a relatada nas estatísticas internacionais. Aventou-se, como um dos motivos, que essa cidade localiza-se na região de clima tropical do país próxima à linha do Equador e possui população de cor não branca, portanto potencialmente com menor risco de fraturas.

O critério utilizado para definir fratura em nosso estudo foi a redução igual ou superior a $20 \%$ na altura do corpo vertebral, em comparação com a vértebra adjacente, mediante análise visual, sem medidas diretas. Este talvez tenha sido um fator de confusão para comparação de prevalências entre diferentes estudos, já que métodos distintos para definição de fratura podem ter sido utilizados 7,14,15. Além disso, em determinados trabalhos, há a opção de não se avaliar a vértebra L5 14, provavelmente por causa da dificuldade de análise visual desta; alguns autores referem risco de um falso diagnóstico de fratura induzido pela presença de alterações degenerativas 28 . Porém, Grados et al. 29 recentemente publicaram um estudo com revisão da literatura e avaliação de experts sobre definição e metodologia para diagnóstico de fratura vertebral e observaram o contrário. Embora não haja consenso sobre o tema, o método semiquantitativo de Genant foi descrito como o de maior utilidade para estudos epidemiológicos e ensaios clínicos 
por ter boa reprodutibilidade e capacidade de distinção entre fraturas e outras deformidades, quando efetuado por analisador treinado. Para reduzir erros de diagnóstico, estabelecemos uma avaliação comparativa entre dois radiologistas capacitados, tendo sido demonstrado alto índice de concordância tanto para coluna torácica, quanto para lombar $(\mathrm{k}=0,89)$.

Nosso questionário incluiu diversos fatores clínicos de risco para fraturas, mas a prevalência do uso de glicocorticóides por tempo superior a três meses e o baixo consumo de cálcio alimentar foram fatores que chamaram nossa atenção pela freqüência de uso divergente da encontrada em estudos prévios. No presente estudo, o uso de glicocorticóides foi superior ao descrito na pesquisa de LAVOS para a população brasileira $(3,4 \%)$ e até mesmo colombiana (5,3\%), a maior referência de usuários daquela investigação 14. Quanto ao consumo alimentar de cálcio, não obstante a orientação padrão ser acima de $1.200 \mathrm{mg} /$ dia, o fato de nenhuma mulher ter referido consumo superior a $600 \mathrm{mg} / \mathrm{dia}$ foi fator de surpresa, principalmente levando-se em conta o PIB per capita da cidade, superior ao da maioria dos municípios brasileiros. Mesmo com essa deficiência alimentar e com história de fraturas prévias por fragilidade, apenas uma pequena parcela da população fazia uso de suplementos de cálcio.

O percentual de consumo de álcool em nossa população, maior do que o referido em outro estudo nacional 14 , poderia ser explicado pelo hábito secular de populações de origem italiana e alemã ingerirem bebidas alcoólicas regularmente. Por outro lado, o grupo investigado nesta pesquisa possui baixo percentual de sedentárias, o que teria alguma influência protetora no que se refere ao risco de fraturas. Altas taxas de atividade física também foram relatadas por Clark et al. 14 sobre a população Argentina. O exercício físico está relacionado ao aumento da resistência muscular, da coordenação motora, do equilíbrio e da flexibilidade, contribuindo para a redução da perda óssea e melhora da saúde em geral 30, bem como para a redução do risco de fraturas de fêmur 31 . No presente estudo, esse fato pode ser explicado pelo fato de que, no município de Chapecó, houve adoção de programas públicos de promoção e prevenção à saúde do idoso nos últimos anos, incluindo atividade física e orientações de saúde periódicas.

A freqüência de história de fratura por fragilidade e de fraturas de coluna identificadas no RX aumentou com a idade, chegando a $60 \%$ na população acima de 80 anos. Bandeira \& Carvalho 15 , em hospital de referência em Pernambuco, avaliaram 174 mulheres com prevalência de fraturas reconhecidas no RX inferior à observada neste estudo. Também observaram que as mulheres com fratura eram mais velhas e com maior tempo de menopausa do que as sem fraturas e que a freqüência aumentava com a idade $(81,8 \%$ após 80 anos). Além disso, verificaram que 87,5\% eram assintomáticas, $28,1 \%$ eram múltiplas e que a localização mais freqüente era no final da coluna torácica, dados semelhantes aos descritos na presente investigação.

As mulheres avaliadas neste estudo também referiram maior prevalência de história familiar de fratura do que o descrito previamente para populações brasileiras 14,15 , mas semelhante ao verificado para mulheres com fratura por fragilidade já estabelecida 11. Esse dado corrobora a idéia de que nossa população parece ter maior risco para fraturas do que o restante da população do país.

$\mathrm{Na}$ análise ajustada, observamos que, a cada dez anos de faixa etária, havia aumento na prevalência de fraturas vertebrais, reforçando a associação entre fraturas e idade já descrita anteriormente 10,11,14. O sedentarismo foi associado a quase o dobro de prevalência de fratura vertebral. Feskanich et al. 31 já haviam relatado associação entre atividade física no período de lazer e risco de fratura.

Apesar de o estudo seccional não ser estratégia para testar hipóteses sobre vínculo causal, é possível testar a existência de associações entre doença/agravo e exposição. Para isso, optamos por apresentar as razões de risco através das razões de prevalência, uma vez que as razões de chance tendem a superestimar as associações com prevalência elevada 32 , como em nosso estudo. Por outro lado, não se pode descartar o efeito do viés de sobrevivência, já que, como apenas um período curto de tempo é avaliado, não se têm informações sobre diferenciais de sobrevivência entre expostos e não expostos 32 . Uma possível limitação desta pesquisa foi o $n$ obtido, pois dificulta a demonstração de efeitos de pequena intensidade. Provavelmente, com um $n$ maior teríamos redução no IC95\% de algumas associações e talvez algumas relações limítrofes fossem significativas.

Em síntese, o presente estudo demonstrou que, em regiões ao sul do Brasil, parece haver uma alta prevalência de fraturas vertebrais. Pelas características geográficas, culturais e climáticas da região, já esperávamos que o resultado fosse diferente do até então relatado em outros estudos nacionais. Tanto a alta prevalência como a variação regional reforçam a idéia da necessidade de políticas públicas adaptáveis para cada população.

O último guia para prevenção e tratamento publicado pela National Osteoporosis Founda- 
tion 12 já orienta o início de tratamento para aumentar a densidade óssea em pacientes que tenham evidência clínica ou morfométrica de fratura de coluna ou quadril, independentemente dos resultados da densitometria óssea. No Brasil, essas orientações não foram ainda amplamente adotadas na prática.

A ocorrência de fraturas vertebrais é a mais comum e precoce relacionada à osteoporose, representando um risco aumentado para a ocorrência de nova fratura vertebral ${ }^{3}$, de fraturas de quadril e de antebraço ${ }^{4}$, de desabilidades diárias $2,4,6$ e de redução na qualidade de vida 31 . Contudo, levando-se em conta que a presença des- sas fraturas é facilmente identificada em exame complementar de baixo custo e risco (radiografia de coluna), sugerimos a realização de radiografia simples de coluna torácica e lombar para mulheres a partir dos 60 anos como método para prevenção secundária de agravos relacionados a fraturas por fragilidade óssea. A radiografia é aconselhável também em regiões onde a realização de rastreamento mais precoce por outros métodos for dificultada pelo valor do exame ou pela distância do centro de referência, devendose iniciar tratamento adequado se for constatada a doença.

\section{Resumo}

Fraturas são o principal agravo relacionado à fragilidade óssea na pós-menopausa, representando aumento de risco de novas fraturas, mortalidade e custos. Nosso objetivo foi verificar a ocorrência de fratura vertebral por fragilidade e relacioná-la com fatores demográficos, comportamentais e clínicos em uma população brasileira. Foi feito um estudo transversal com seleção por amostragem aleatória estratificada de mulheres idosas residentes em Chapecó, Santa Catarina, Brasil. A amostra foi constituída por 186 mulheres brancas com idade acima de 60 anos. Destas, 48,9\% tinham fraturas vertebrais assintomáticas, com maiores prevalências em T11-12 e L4-5. Na análise ajustada, observa-se que existe gradiente entre idade e fratura vertebral, chegando a ser 2,3 vezes maior a prevalência de fraturas entre as mulheres com idade superior a 80 anos. As sedentárias apresentaram prevalência de fratura 1,44 vez maior do que as não sedentárias. Devido à alta prevalência de fraturas vertebrais encontrada, sugerimos a realização de radiografia de coluna para mulheres idosas para rastreamento e prevenção de agravos.

Fraturas da Coluna Vertebral; Osteoporose; Pós-Menopausa; Saúde da Mulher 


\section{Colaboradores}

P. P. Oliveira foi responsável pelo desenho do projeto, análise e interpretação dos dados, levantamento bibliográfico e redação do artigo. L. P. F. Marinheiro e M. C. O. Wender contribuíram no desenho do projeto, discussão dos resultados, desenho e redação do artigo. F. Roisenberg e P. G. S. Lacativa colaboraram na análise e interpretação dos dados, revisão crítica, discussão dos resultados, desenho e redação do artigo.

\section{Agradecimentos}

Agradecemos pelo esforço e dedicação de toda a equipe de coleta de dados, composta por estudantes de medicina da Universidade Comunitária da Região de Chapecó: C. Sandrin, G. Morandini, J. B. Mendes, J. K. Grellman, K. Bigolin, M. G. Lang, P. H. Batista, V. Gheno, que participaram deste projeto com muito empenho e dedicação. Também agradecemos ao essencial apoio de D. Benzano na revisão da análise estatística.

\section{Referências}

1. Kanis JA, Johnell O, Oden A, Sembo I, RedlundJohnell I, Dawson A, et al. Long-term risk of osteoporotic fracture in Malmö. Osteoporos Int 2000; 11:669-74

2. Papaioannou A, Watts NB, Kendler DL, Yuen CK, Adachi JD, Ferko N. Diagnosis and management of vertebral fractures in elderly adults. Am J Med 2002; 113:220-8.

3. Lindsay R, Silverman SL, Cooper C. Risk of new vertebral fracture in the year following a fracture. JAMA 2001; 285:320-3.

4. Naves M, Díaz-López JB, Gómez C, Rodríguez-Rebollar A, Rodríguez-García M, Cannata-Andía JB. The effect of vertebral fracture as a risk factor for osteoporotic fracture and mortality in a Spanish population. Osteoporos Int 2003; 14:520-4.

5. Genant HK, Li J, Wu CY, Shepherd JA. Vertebral fractures in osteoporosis: a new method for clinical assessment. J Clin Densitom 2000; 3:281-90.

6. Cauley JA, Thompson DE, Ensrud KC, Scott JC, Black D. Risk of mortality following clinical fractures. Osteoporos Int 2000; 11:556-61.

7. Pinheiro MM, Castro CM, Szejnfeld VL. Low femoral bone mineral density and quantitative ultrasound are risk factores for new osteoporotic fracture and total and cardiovascular mortality: a 5-year population-based study of Brazilian elderly women. J Gerontol A Biol Sci Med Sci 2006; 61:196-203.
8. Black DM, Steinbuch M, Palermo L, Dargent-Molina P, Lindsay R, Hoseyni MS, et al. An assessment tool for predicting fracture risk in postmenopausal women. Osteoporos Int 2001; 12:519-28.

9. Henry MJ, Pasco JA, Sanders KM, Nicholson GC, Kotowicz MA. Fracture Risk (FRISK) Score: Geelong Osteoporosis Study. Radiology 2006; 241:190-6.

10. Nguyen TV, Center JR, Pocock NA, Eisman JA. Limited utility of clinical indices for the prediction of symptomatic fracture risk in postmenopausal women. Osteoporos Int 2004; 15:49-55.

11. Pinheiro MM, Ciconelli RM, Martini LA, Ferraz MB. Clinical risk factors for osteoporotic fractures in Brazilian women and men: the Brazilian Osteoporosis Study (BRAZOS). Osteoporos Int 2009; 20:399-408.

12. National Osteoporosis Foundation. Clinician's guide to prevention and treatment of osteoporosis. Washington DC: National Osteoporosis Foundation; 2008.

13. Castro da Rocha FA, Ribeiro AR. Low incidence of hip fractures in an equatorial area. Osteoporos Int 2003; 14:496-9.

14. Clark P, Cons-Molina F, Ragi S, Deleze M, Haddock $\mathrm{L}$, Zanchetta J, et al. The prevalence of vertebral fractures in Latin American countries: the Latin American Vertebral Osteoporosis Study "LAVOS". Osteoporos Int 2009; 20:275-82. 
15. Bandeira FA, Carvalho EF. Prevalência de osteoporose e fraturas vertebrais em mulheres na pósmenopausa atendidas em serviços de referência. Rev Bras Epidemiol 2007; 10:86-98.

16. Silveira VA, Medeiros MMC, Coelho-Filho JM, Mota RS, Noleto JCS, Costa FS, et al. Incidência de fratura do quadril em área urbana do Nordeste brasileiro. Cad Saúde Pública 2005; 21:907-12.

17. Komatsu RS, Ramos LR, Szejnfeld VL. Incidence of proximal femur fractures in Marilia, Brazil. J Nutr Health Aging 2004; 8:362-7.

18. Clark P, Cons-Molina F, Ragi S, Deleze M, Haddock $\mathrm{L}$, Zanchetta J, et al. The prevalence of vertebral fractures in Latin American women: The Latin American Vertebral Osteoporosis Study "LAVOS". Osteoporos Int 2006; 17 Suppl 2:S130-42.

19. O'Neill TW, Cooper C, Algra D, Pols HAP, Agnusdei D, Dequeker J, et al. Design and development of a questionnaire for use in a multicentre study of vertebral osteoporosis in Europe: The European Vertebral Osteoporosis Study (EVOS). Rheumatology in Europe 1995; 24:75-81.

20. Calle EE, Thun MJ, Petrelli JM, Rodriguez C, Heath Jr. CW. Body-mass index and mortality in a prospective cohort of U.S. adults. N Engl J Med 1999; 341:1097-105.

21. Núcleo de Estudos e Pesquisas em Alimentação. Tabela brasileira de composição de alimentos. 2a Ed. Campinas: Núcleo de Estudos e Pesquisas em Alimentação; 2006.

22. Ferreira MG, Valente JG, Gonçalves-Silva RMV, Sichieri R. Acurácia da circunferência da cintura e da relação cintura/quadril como preditores de dislipidemias em estudo transversal de doadores de sangue de Cuiabá, Mato Grosso, Brasil. Cad Saúde Pública 2006; 22:307-14.

23. Genant HK, Wu CY, van Kuijk C, Nevitt MC. Vertebral fracture assessement using a semiquantitaive technique. J Bone Miner Res 1993; 8:1137-48.
24. Instituto Brasileiro de Geografia e Estatística. Indicadores sociais, 2003. Rio de Janeiro: Instituto Brasileiro de Geografia e Estatística; 2004.

25. Ramalho AC, Lazaretti-Castro M, Hauache O, Vieira JG, Takata E, Cafalli F, et al. Osteoporotic fractures of proximal femur: clinical and epidemiological features in a population of the city of São Paulo. Sao Paulo Med J 2001; 119:48-53.

26. O'Neill TW, Felsenberg D, Varlow J, Cooper C, Kanis JA, Silman AJ. The prevalence of vertebral deformity in European men and women: the European Vertebral Osteoporosis Study. J Bone Miner Res 1996; 11:1010-8.

27. Johnell O, Borgstrom F, Jonsson B, Kanis J. Latitude, socioeconomic prosperity, mobile phones and hip fracture risk. Osteoporos Int 2007; 18:333-7.

28. Jones G, White C, Nguyen T, Sambrook PN, Kelly PJ, Eisman JA. Prevalent vertebral deformities: relationship to bone mineral density and spinal osteophytosis in elderly men and women. Osteoporos Int 1996; 6:233-9.

29. Grados F, Fechtenbaum J, Flipon E, Kolta S, Roux C, Fardellone P. Radiographic methods for evaluating osteoporotic vertebral fractures. Joint Bone Spine 2009; 76:241-7.

30. Chan KM, Anderson M, Lau EMC. Exercise interventions: defusing the world's osteoporosis time bomb. Bull World Health Organ 2003; 81:827-30.

31. Feskanich D, Willet W, Colditz G. Walking and leisure-time activity and risk of hip fracture in postmenopausal women. JAMA 2002; 288:2300-6.

34. Coutinho LMS, Scazufca M, Menezes PR. Métodos para estimar razão de prevalência em estudos de corte transversal. Rev Saúde Pública 2008; 42:992-8.

Recebido em 27/Set/2009

Versão final reapresentada em 30/Mar/2010

Aprovado em 10/Jun/2010 